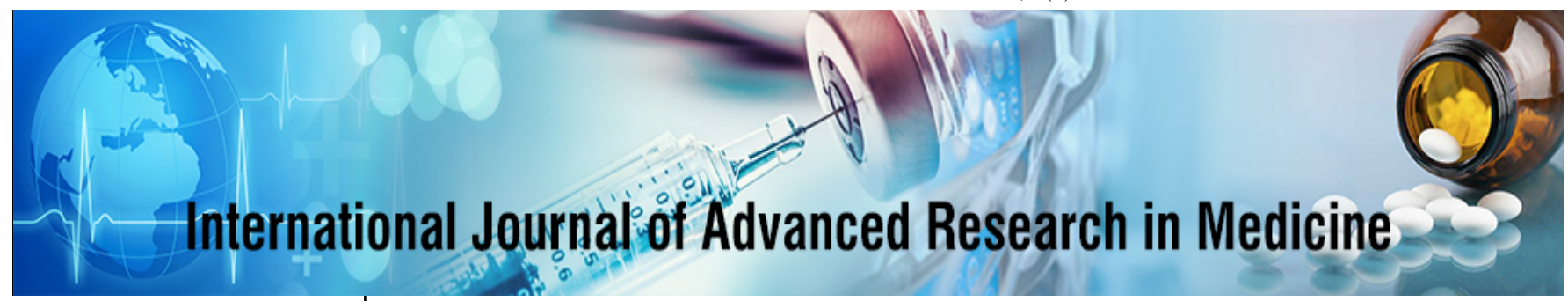

E-ISSN: 2706-9575

P-ISSN: 2706-9567

IJARM 2021; 3(2): 432-434

Received: 25-06-2021

Accepted: 05-08-2021

Dr. Balasaheb G Shelke Assistant Professor, SSPM Medical College \& Lifetime Hospital, Padve, Maharashtra, India
Corresponding Author: Dr. Balasaheb G Shelke Assistant Professor, SSPM Medical College \& Lifetime Hospital, Padve, Maharashtra, India

\section{COVID-19 in tuberculosis in patients}

\author{
Dr. Balasaheb G Shelke
}

DOI: https://doi.org/10.22271/27069567.2021.v3.i2g.287

Abstract

Coronavirus Disease 2019 (COVID-19) has become a pandemic and the number of infected cases continues to rise. Millions have been laboratory-confirmed cases and reported worldwide, with a mortality rate of $4.6 \%$. To be the best of our knowledge, study not has yet evaluated the impact of tuberculosis on COVID-19 severity and mortality in India. Therefore, we performed a study to assess whether biochemical values in tuberculosis is more deranged when compared to the person who is not having the tuberculosis and suffering from the infection of COVID-19.

Keywords: Tuberculosis, covid-19, biochemical parameters

\section{Introduction}

Coronavirus Disease 2019 (COVID-19) has become a pandemic and the number of infected cases continues to rise. Millions have been laboratory-confirmed cases and reported worldwide, with a mortality rate of $4.6 \%{ }^{[1]}$. To be the best of our knowledge, study not has yet evaluated the impact of tuberculosis on COVID-19 severity and mortality in India. Previous meta-analyses in other countries have shown that chronic obstructive pulmonary disease (COPD), cerebrovascular disease, hypertension, diabetes, and cardiovascular disease were risk factors for disease progression in patients with COVID-19 ${ }^{[2-6]}$. However, to be the best of our knowledge, study has yet evaluated the impact of tuberculosis on COVID-19 severity and mortality. Therefore, we performed a study to assess whether biochemical values in tuberculosis is more deranged when compared to the person who is not having the tuberculosis and suffering from the infection of COVID-19.

\section{Aims and Objectives}

To study and find out whether there are any difference between the biochemical markers in tuberculosis with Covid infections and without Tuberculosis.

\section{Materials and Methods}

This study was done in the Department of Pulmonology, SSPM Medical College \& Lifetime Hospital Padve. The study was done from April 2020 to March 2021. Thirty cases were chosen out of which fifteen were confirmed to have tuberculosis with T.B and the other fifteen were only covid positive cases.

\section{Exclusion criteria}

TB with other co-morbidities, so that there was no bias. All thirty were not known to have any co-morbidities.

\section{Results}

Table 1: Mean Values (rounded off to nearest one decimal point) disorder, anxiety

\begin{tabular}{|c|c|c|}
\hline Markers & Without TB & With TB \\
\hline Serum sodium, mmol/L & 137 & 141 \\
\hline Serum potassium, mmol/L & 4 & 3.8 \\
\hline Serum chloride, mmol/L & 96 & 94 \\
\hline AST U/L & 69 & 96 \\
\hline ALT U/L & 38 & 127 \\
\hline LDH U/L & 682 & 1300 \\
\hline CRP, mg/L & 35 & 117 \\
\hline D-dimer, ng/mL & 670 & 900 \\
\hline Ferritin ng/ml & 260 & 9 \\
\hline
\end{tabular}




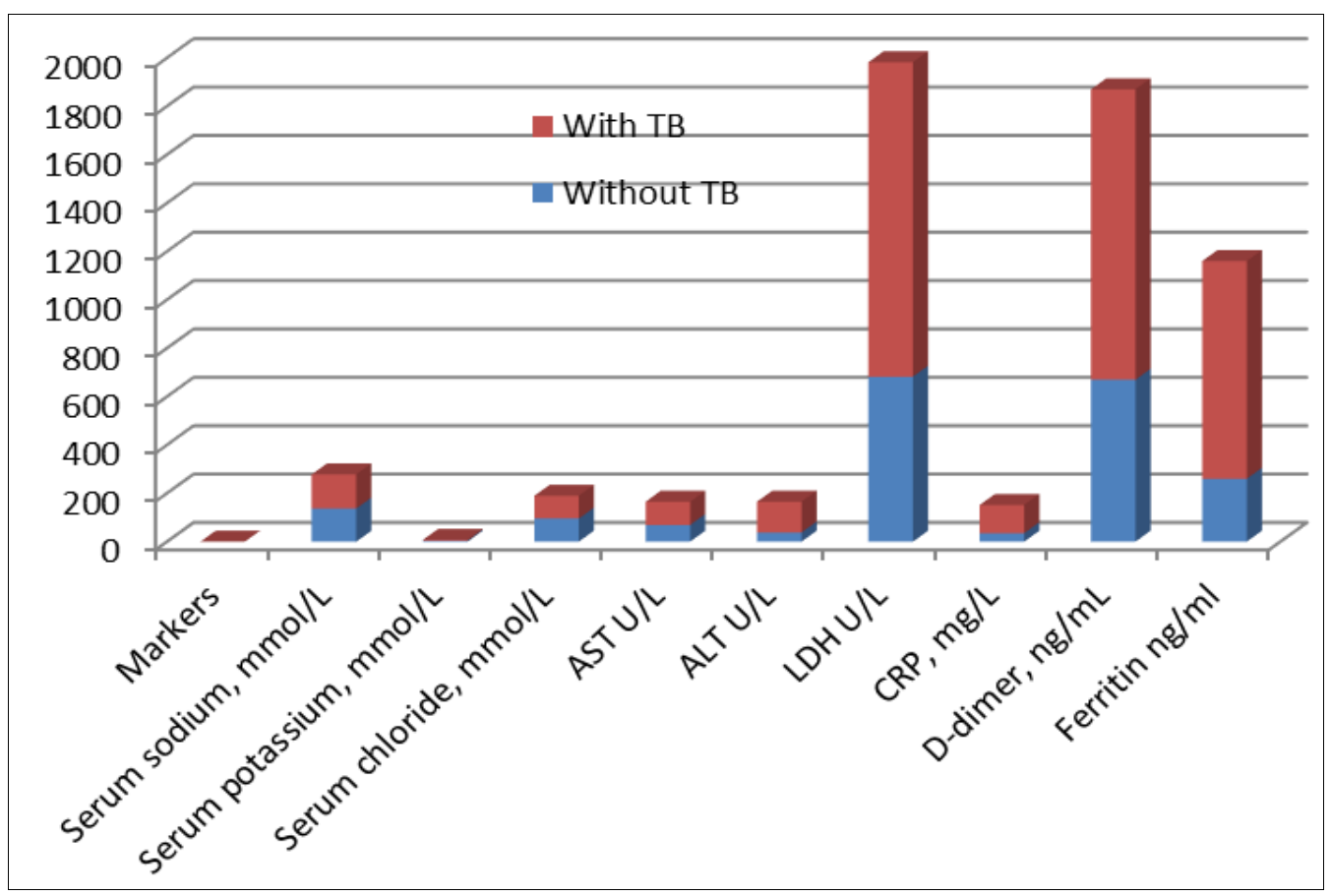

Graph 1: Mean Values (rounded off to nearest one decimal point)

\section{Discussion}

Having a reliable estimate of the association between tuberculosis and COVID-19 severity and mortality is crucial to ensure specific successful global preventive and treatment strategies for tuberculosis patients. Our study revealed that tuberculosis was associated with a ${ }^{[2]} 10$-fold increased risk of severe COVID-19 disease, although the statistical difference was not significant. When a patient suffers from a previous respiratory disease, the patient's lung function is impaired, and their resistance to viruses is low and they tend to develop ARDS ${ }^{[2]}$. Therefore, tuberculosis may be a risk factor for disease progression. This study highlights the need for effective preventive measures and treatment strategies to reduce the risk of COVID-19 severity in tuberculosis patients. However, our study did not suggest that tuberculosis was associated with an increased risk of mortality. This may be due to the small number of samples used in the analysis. Further analysis is needed to validate this result in the future. Four studies ${ }^{[7-10]}$ involving 2, 383 patients reported the prevalence of tuberculosis between severe and non-severe COVID-19 patients. The meta-analysis indicated the prevalence of tuberculosis in severe patients $(1.47 \%, 10 / 680)$ was higher than that in non-severe patients $(0.59 \% ; 10 / 1703)(\mathrm{OR}=2.10,95 \% \mathrm{CI}$ : 0.61 to $7.18 ; P=.24 ; I^{2}=36 \%$ ), although the statistical difference was not significant. The univariate meta-regression analysis indicated the mean age of patients was not the source of heterogeneity or the factor affecting the correlation between tuberculosis and COVID-19 severity. Two studies [11, 12] provided the prevalence of tuberculosis between surviving and dying COVID-19 patients. Chen et al. study ${ }^{[11]}$ revealed that non-survivors had a higher prevalence of tuberculosis than survivors (5.26\% vs. 0\%). However, Du et al. study ${ }^{[12]}$ showed non-survivors had a lower prevalence of tuberculosis than survivors (0\% vs 5.06\%). These inconsistent results may be due to differences in the follow-up time of included studies and the treatment regimens of the patients.

\section{Conclusion}

The biochemical markers are more deranged in tuberculosis when compared to people without tuberculosis. Other comorbidities were not included so that it did not formed a bias in the study.

\section{References}

1. World Health Organization. Coronavirus disease (COVID-2019) situation reports. Accessed July 9, 2020.

2. Zheng Z, Peng F, Xu B, et al. Risk factors of critical \& mortal COVID-19 cases: a systematic literature review and meta-analysis. J Infect, 2020.

3. Wang B, Li R, Lu Z, Huang Y. Does comorbidity increase the risk of patients with COVID-19: evidence from meta-analysis. Aging. 2020;12(7):6049-6057.

4. Moher D, Liberati A, Tetzlaff J, Altman DG. Preferred reporting items for systematic reviews and meta-analyses: the PRISMA statement BMJ (Clinical research ed). 2009;339:b2535.

5. Gao Y, Chen Y, Liu M, Shi S, Tian J. Impacts of immunosuppression and immunodeficiency on COVID-19: systematic review and meta-analysis. J Infect, 2020. 10.1016/j.jinf.2020.05.017.

6. Liu M, Gao Y, Shi S, Chen Y, Yang K, Tian J. Drinking no-links to the severity of COVID-19: a systematic review and meta-analysis. J Infect, 2020. 10.1016/j.jinf.2020.05.042

7. Li X, Xu S, Yu M, et al. Risk factors for severity and mortality in adult COVID-19 inpatients in Wuhan. J Allergy Clin Immunol. 2020;146(1):110-118.

8. Liu SJ, Cheng F, Yang XY, et al. A study of laboratory confirmed cases between laboratory indexes and clinical classification of 342 cases with corona virus disease 2019 in Ezhou. Lab Med, 2020. 10.3969/j.issn.1673-8640.2019.00.000

9. Xiao KH, Shui LL, Pang $\mathrm{XH}$, et al. The clinical features of the 143 patients with COVID-19 in 
North-East of Chongqing. J Third Milit Med Univ., 2020. 10.16016/j.1000-5404.202002097

10. Zhang YT, Deng AP, Hu T, et al. Clinical outcomes of COVID-19 cases and influencing factors in Guangdong province. Zhonghua Liu Xing Bing Xue Za Zhi. 2020;41:E057.

11. Chen T, Dai Z, Mo P, et al. Clinical characteristics and outcomes of older patients with coronavirus disease 2019 (COVID-19) in Wuhan, China (2019): a single-centered, retrospective study. J Gerontol A Biol Sci Med Sci. 2020. 10.1093/gerona/

12. Du RH, Liang LR, Yang CQ, et al. Predictors of mortality for patients with COVID-19 pneumonia caused by SARS-CoV-2: a prospective cohort study. Eur Respir J. 2020;55(5):2000524. 